\title{
Effect of Internal Optical Loss on Threshold Characteristics of Semiconductor Lasers With a Quantum-Confined Active Region
}

\author{
Levon V. Asryan and Serge Luryi, Fellow, IEEE
}

\begin{abstract}
We develop a general approach to including the internal optical loss in the description of semiconductor lasers with a quantum-confined active region. We assume that the internal absorption loss coefficient is linear in the free-carrier density in the optical confinement layer and is characterized by two parameters, the constant component and the net cross section for all absorption loss processes. We show that, in any structure where the free-carrier density does not pin in the presence of light generation, the free-carrier-density dependence of internal loss gives rise to the existence of a second lasing threshold above the conventional threshold. Above the second threshold, the light-current characteristic is two-valued up to a maximum current at which the lasing is quenched. We show that the presence of internal loss narrows considerably the region of tolerable structure parameters in which the lasing is attainable; for example, the minimum cavity length is significantly increased. Our approach is quite general but the numerical examples presented are specific for quantum dot (QD) lasers. Our calculations suggest that the internal loss is likely to be a major limiting factor to lasing in short-cavity QD structures.
\end{abstract}

Index Terms-Quantum dots (QDs), quantum wells (QWs), quantum wires (QWRs), semiconductor heterojunctions, semiconductor lasers.

\section{INTRODUCTION}

I NTERNAL optical loss is present in all types of semiconductor lasers. It adversely affects their operating characteristics - increasing the threshold current density and decreasing the differential efficiency [1]-[3]. Because of the lower value of the optical confinement factor for thin layers, the effect of internal loss is stronger for lasers with a reduced-dimensionality active region than for bulk lasers [1].

In general, several mechanisms can contribute to the internal loss, such as free-carrier absorption in the optical confinement layer (OCL) and in the cladding layers (emitters) [4], intervalence band absorption (hole photoexcitation into the split-off subband) [5]-[8], carrier absorption in the quantum-confined active region itself, and scattering at rough surfaces and imperfections of the waveguide. Determination of the absorption co-

Manuscript received January 26, 2004; revised April 2, 2004. This work was supported in part by the Air Force Office of Scientific Research Multidisciplinary University Research Initiative Program, Award no. F49620-00-1-0331, managed by T. Steiner and in part by the New York State Center for Advanced Sensor Technology.

L. V. Asryan is with the State University of New York at Stony Brook, Stony Brook, NY 11794-2350 USA, on leave from the Ioffe Physico-Technical Institute, St. Petersburg 194021, Russia (e-mail: asryan@ece.sunysb.edu).

S. Luryi is with the State University of New York at Stony Brook, Stony Brook, NY 11794-2350 USA (e-mail: Serge.Luryi@sunysb.edu).

Digital Object Identifier 10.1109/JQE.2004.830207 efficient for each of these processes is very important because, depending on their relative strengths and the structure design parameters, the net absorption loss coefficient can be as low as $1.4 \mathrm{~cm}^{-1}$ [9] or as high as $20 \mathrm{~cm}^{-1}$ [10], and even higher [11].

Due to the variety of possible mechanisms, one hardly expects a first-principle evaluation of the net internal loss coefficient. Formally, however, all different processes can be grouped into two categories, one dependent on the injection carrier density (such as free-carrier absorption in the OCL), the other insensitive to this density (such as scattering at rough interfaces).

Leaning upon this fact, we develop here a general phenomenological approach to the inclusion of the effect of internal loss on threshold characteristics in semiconductor lasers. We show that the injection-carrier-density dependence of internal loss coefficient, combined with nonpinning of the carrier density, gives rise to the existence of a second lasing threshold above the conventional threshold; above the second threshold, the light-current characteristic is two-valued. We also show that the presence of internal loss narrows considerably the region of tolerable structure parameters in which the lasing is attainable.

The total net internal loss coefficient (which we shall refer to as the internal loss) is presented as the sum of a constant $\alpha_{0}$ and a component linear in the carrier density in the OCL $n$ as follows:

$$
\alpha_{\text {int }}=\alpha_{0}+\sigma_{\text {int }} n
$$

where $\sigma_{\text {int }}$ can be viewed as an effective cross section for all absorption loss processes.

The assumption of a linear dependence on the free-carrier density in the waveguide is justified in most situations of practical interest. For example, intervalence band absorption increases proportionally to hole density [5]-[7]; free-carrier absorption also increases linearly with $n$ [4].

The carrier densities in the cladding layers, being mainly defined by the doping levels there, remain practically unchanged and close to their built-in values as the injection current varies. ${ }^{1}$ For this reason, the free-carrier and the intervalence band absorption loss due to the optical mode penetration into the cladding layers are both lumped into the constant component $\alpha_{0}$ of the internal loss.

\footnotetext{
${ }^{1}$ There may be a slight increase in the carrier density above their built-in values in the cladding layers due to the carrier leakage from the OCL at high injection level and high temperature. In the first approximation, this variation should also linearly follow the carrier density in the OCL $n$. Hence, the inclusion of this effect will slightly increase the value of $\sigma_{\text {int }}$ in (1).
} 


\section{LASING THRESHOLD CONDITION}

With (1), the lasing threshold condition (balance between the modal gain $g=g^{\max }\left(f_{n}+f_{p}-1\right)$ and the total loss $\left.\beta+\alpha_{\text {int }}\right)$ becomes

$$
g^{\max }\left(f_{n}+f_{p}-1\right)=\beta+\alpha_{0}+\sigma_{\text {int }} n
$$

where $g^{\max }$ is the maximum (saturation) value of the modal gain, $\beta=(1 / L) \ln (1 / R)$ is the external (mirror) loss, $L$ is the cavity length, and $R$ is the mirror reflectivity. In (1) and (2), $\alpha_{\text {int }}$ is the weighted average of the internal loss across the optical mode shape [3].

For quantum-well (QW) or quantum-wire (QWR) lasers, $f_{n}$ and $f_{p}$ are occupancies of the electron and hole subband-edge levels, between which the lasing transitions occur. For a quantum-dot (QD) laser, $f_{n}$ and $f_{p}$ are occupancies of the discrete electron and hole levels. The maximum value $g^{\max }$ of the modal gain $g$ is obtained at full occupancies $f_{n}=f_{p}=1$ and the minimum $g=-g^{\max }$ at zero occupancies.

For QW or QWR lasers, the right-hand sides of (1) and (2) should also contain a term for absorption in the active region, which is linear in the two-dimensional (2-D) or one-dimensional (1-D) carrier density, respectively. However, at high injection currents (or high temperatures-see [12] and [13]), this term will be small compared to absorption in the OCL.

In a QD laser, the process analogous to free-carrier absorption is carrier photoexcitation from the QD levels to states in the continuous spectrum [14], [15]. The absorption coefficient for this process is linear in the confined-carrier level occupancy in a QD and, generally, it should also be included into the right-hand sides of (1) and (2). However, this contribution is typically less than about $0.1 \mathrm{~cm}^{-1}$ [14], [15].

In general, in the right-hand sides of (1) and (2) one should use separate terms for electrons and holes, since they have different cross sections $\sigma_{\text {int }}^{n}$ and $\sigma_{\text {int }}^{p}$. For simplicity, we will use the lasing threshold condition in the form of (2) having left understood that $\sigma_{\text {int }}$ refers to the cross section corresponding to the carrier type dominant in absorption.

We assume equal electron and hole occupancies in a quantum-confined active region $\left(f_{n}=f_{p}\right)$. At relatively high temperatures and below the lasing threshold, the thermal equilibrium holds and $f_{n}$ is given by the Fermi-Dirac distribution function with the quasi-Fermi level determined by the pumping. ${ }^{2}$ The carrier density $n$ in the waveguide (OCL) is related to $f_{n}$ as follows [14]:

$$
n=n_{1} \frac{f_{n}}{1-f_{n}}
$$

where $n_{1}=N_{c}^{\mathrm{OCL}} \exp \left(-E_{n} / T\right)$ is a quantity characterizing the intensity of thermally excited escape of carriers from a re-

\footnotetext{
${ }^{2}$ It was shown in [14] that, depending on temperature and carrier localization energies, two fundamentally different modes of QD filling with carriers, nonequilibrium and equilibrium, are possible. At low $T$ (nonequilibrium QD filling), the threshold current is virtually temperature independent; at high $T$ (equilibrium filling), the threshold current is controlled by thermal escape from QDs and grows exponentially with $T$. Since the carrier density in the OCL is low at low $T$, the effect of carrier-density-dependent internal loss is weak in the nonequilibrium mode.
}

duced-dimensionality active region to the OCL, with $N_{c}^{\mathrm{OCL}}=$ $2\left(m_{c}^{\mathrm{OCL}} T / 2 \pi \hbar^{2}\right)^{3 / 2}, E_{n}$ is the carrier excitation energy from an active region, and the temperature $T$ is measured in units of energy.

The threshold condition is then written as follows:

$$
g^{\max }\left(2 f_{n}-1\right)=\frac{1}{L} \ln \frac{1}{R}+\alpha_{0}+\sigma_{\text {int }} n_{1} \frac{f_{n}}{1-f_{n}} .
$$

It is illustrated in Fig. 1(a) where the modal gain $g=g^{\max }\left(2 f_{n}-1\right)$ and the internal loss $\alpha_{\text {int }}=$ $\alpha_{0}+\sigma_{\text {int }} n_{1} f_{n} /\left(1-f_{n}\right)$ are shown as functions of the level occupancy $f_{n}$. Though the theoretical approach developed here is general and applies equally to semiconductor lasers with a quantum-confined active region of an arbitrary dimensionality, our numerical examples, including those in Figs. 1-8, are specific for QD lasers; the simulation parameters are given in Section $\mathrm{V}$-A.

With (3), the level occupancy in the active region and the modal gain can be expressed in terms of the carrier density in the OCL as follows:

$$
\begin{aligned}
f_{n} & =\frac{n}{n+n_{1}} \\
g & =g^{\max } \frac{n-n_{1}}{n+n_{1}} .
\end{aligned}
$$

The threshold condition becomes

$$
g^{\max } \frac{n-n_{1}}{n+n_{1}}=\frac{1}{L} \ln \frac{1}{R}+\alpha_{0}+\sigma_{\text {int }} n
$$

Fig. 1(b), showing the modal gain and the internal loss as functions of the carrier density in the OCL $n$ (given by (6) and (1), respectively), illustrates the threshold condition of the form (7).

In the absence of lasing, the injection current density $j$ is related to the level occupancy in the active region $f_{n}$ as follows [14], [16]:

$$
j=j_{\text {spon }}^{\text {active }}+e b B n^{2}=j_{\text {spon }}^{\text {active }}\left(f_{n}\right)+e b B n_{1}^{2} \frac{f_{n}^{2}}{\left(1-f_{n}\right)^{2}}
$$

where $b$ is the OCL thickness and $B$ is the radiative constant for the OCL. A relation between the spontaneous recombination current density in a quantum-confined active region $j_{\text {spon }}^{\text {active }}$ and the level occupancy is presented in Appendix I.

With the functional relationship (8) between the level occupancy $f_{n}$ and the injection current density $j$, both the modal gain and the internal loss can be calculated as functions of $j$ [shown in Fig. 1(c)].

\section{SOlutions OF THE THRESHOLD CONDITION: TwO LASING THRESHOLDS}

For $\sigma_{\text {int }} \neq 0,(4)$ is a quadratic equation in the confinedcarrier level occupancy in the active region $f_{n}$; the roots are (see Fig. 1 for a graphic illustration to the solutions)

$$
f_{n_{-} \text {th } 1, n_{-} \text {th } 2}=f_{n_{-} \text {th }}^{\text {crit }} \mp \sqrt{\left(f_{n_{-} \text {th }}^{\text {crit }}\right)^{2}-f_{n 0}-\frac{1}{2} \frac{\alpha_{0}}{g^{\max }}}
$$




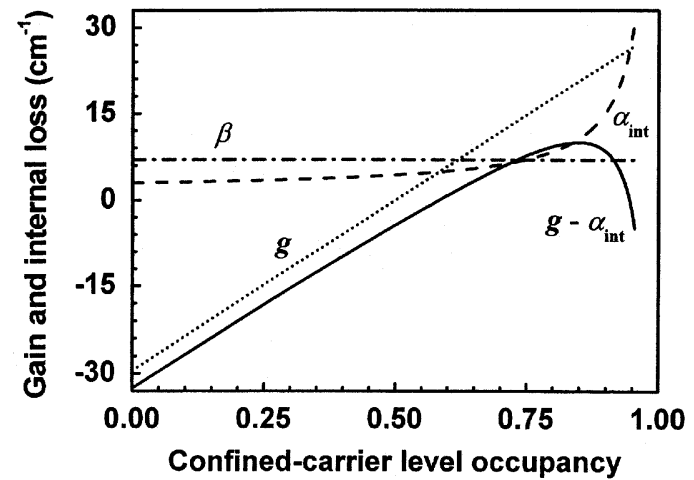

(a)

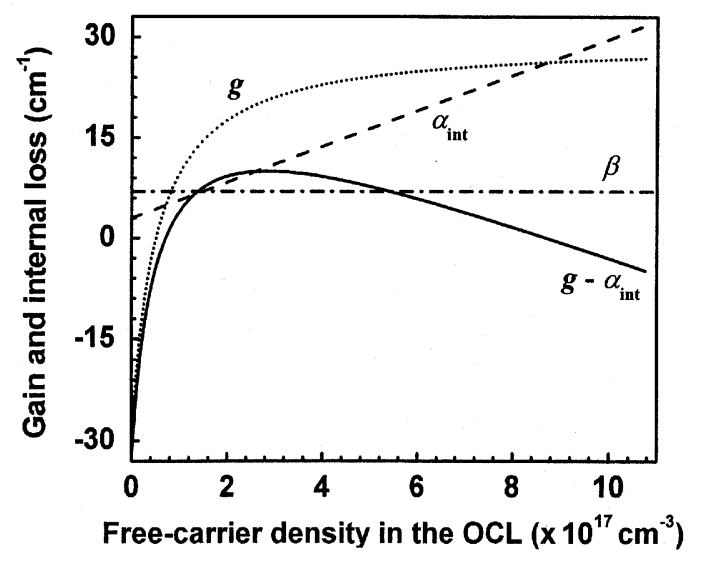

(b)

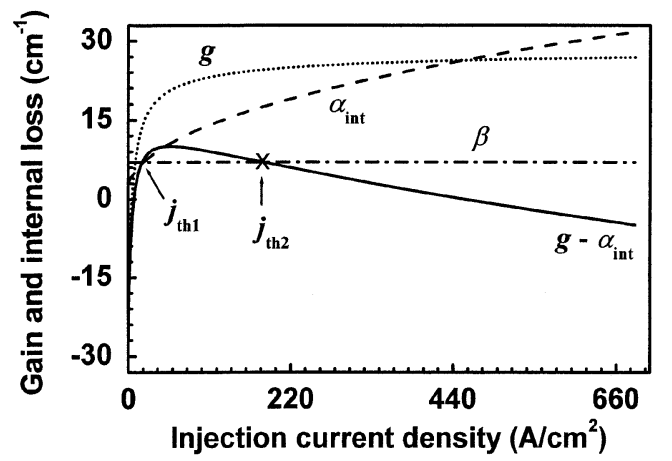

(c)

Fig. 1. Illustration of the threshold condition (4) and of the two lasing thresholds. Modal gain $g=g^{\max }\left(n-n_{1}\right) /\left(n+n_{1}\right)=g^{\max }\left(2 f_{n}-1\right)$ [inclined dotted line in (a) and dotted curves in (b) and (c)], internal loss $\alpha_{\text {int }}=\alpha_{0}+\sigma_{\text {int }} n=\alpha_{0}+\sigma_{\text {int }} n_{1} f_{n} /\left(1-f_{n}\right)$ [dashed curves in (a) and (c) and inclined dashed line in (b)] and difference of modal gain and internal loss (solid curve) against (a) confined-carrier level occupancy in the active region $f_{n}$, (b) free-carrier density in the OCL $n$, and (c) injection current density $j$. The intersections of the solid curve and the horizontal dash-dotted line for the mirror loss $\beta=(1 / L) \ln (1 / R)$ are the solutions (9) of (4) [in (a)], the free-carrier densities in the OCL at the lower and the upper thresholds [in (b)], and the lower and the upper threshold current densities, $j_{\text {th1 }}$ and $j_{\text {th2 } 2}$, respectively [in (c)]. The dependences on $n$ in (b) and on $j$ in (c) are easily converted from those in (a) using (3) and (8). Throughout the paper, a GaInAsP-InP-based QD-heterostructure lasing near $1.55 \mu \mathrm{m}$ [14]-[16] is considered for illustration. We assume 10\% QD-size fluctuations and $N_{S}=6.11 \times 10^{10} \mathrm{~cm}^{-2}$; at these parameters, $g^{\max }=29.52 \mathrm{~cm}^{-1}$. At $T=300 \mathrm{~K}, n_{1}=5.07 \times 10^{16} \mathrm{~cm}^{-3}$. In Figs. 1 and 2, the mirror loss $\beta=7 \mathrm{~cm}^{-1}$; otherwise, $\beta=10 \mathrm{~cm}^{-1}$. Parameters $\alpha_{0}$ and $\sigma_{\text {int }}$ are plausibly taken as $3 \mathrm{~cm}^{-1}$ and $2.67 \times 10^{-17} \mathrm{~cm}^{2}$, respectively.

where

$$
f_{n_{-} \text {th }}^{\text {crit }}=\frac{1}{2}\left(1+f_{n 0}+\frac{1}{2} \frac{\alpha_{0}}{g^{\max }}-\frac{1}{2} \frac{\sigma_{\text {int }} n_{1}}{g^{\max }}\right)
$$

is the "critical" solution [corresponding to the case when a structure parameter attains its critical tolerable value-cf. (17) in Section V], and

$$
f_{n 0}=\frac{1}{2}\left(1+\frac{\beta}{g^{\max }}\right)=\frac{1}{2}\left(1+\frac{L_{0}^{\min }}{L}\right)
$$

is the level occupancy in the active region at the lasing threshold in the absence of internal loss $\left(\alpha_{0}=0, \sigma_{\text {int }}=0\right), L_{0}^{\min }$ being the minimum tolerable cavity length in the absence of internal loss given as

$$
L_{0}^{\min }=\frac{1}{g^{\max }} \ln \frac{1}{R} .
$$

For $L$ shorter than the minimum tolerable cavity length, the lasing is unattainable in the structure. We discuss the minimum cavity length in detail in Section V-B.

In general, the following inequalities hold for $f_{n_{-} \text {th1 }}$ and $f_{n_{-} \text {th2 }}$ (Fig. 1):

$$
\frac{1}{2} \leq f_{n 0} \leq f_{n_{-} \text {th } 1} \leq f_{n_{-} \text {th }}^{\text {crit }} \leq f_{n_{-} \text {th } 2}<1 .
$$

The value $1 / 2$ is the level occupancy at the transparency threshold [when the modal gain is zero: $g^{\max }\left(2 f_{n}-1\right)=0$ ].

Both solutions (9) are physically meaningful and describe two distinct lasing thresholds. The first solution $f_{n_{-} \text {th1 }}$ is the conventional threshold, similar to $f_{n 0}$ but modified by the internal loss. The second solution $f_{n_{-} \text {th2 }}$ appears purely as a consequence of the carrier-density-dependent component of the internal loss in the OCL.

As $\sigma_{\text {int }}$ decreases, the first threshold $f_{n_{-} \text {th1 }}$ decreases and the second threshold $f_{n-t h 2}$ increases [see Fig. 6(b) below]. At $\sigma_{\text {int }}=0$, the only solution of (4) is

$$
f_{n_{-} \mathrm{th} 1}=f_{n 0}+\frac{1}{2} \frac{\alpha_{0}}{g^{\max }}=\frac{1}{2}\left(1+\frac{\beta+\alpha_{0}}{g^{\max }}\right) .
$$

Clearly $f_{n_{-} \text {th } 1}=f_{n 0}$ when both $\alpha_{0}$ and $\sigma_{\text {int }}$ are zero.

Thus, when the internal loss depends on carrier density, there are, in general, two solutions of the threshold condition $f_{n_{-} \text {th1 }}$ and $f_{n_{-} \text {th2 }}$, and hence we have two lasing thresholds.

We shall refer to the injection current densities corresponding to $f_{n_{-} \text {th1 }}$ and $f_{n_{\text {_th2}}}$, respectively, as the lower threshold current

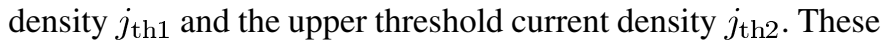
threshold current densities are given by (8) wherein one substitutes either $f_{n}=f_{n \_ \text {th1 }}$ or $f_{n}=f_{n_{-} \text {th2 }}$.

The existence of a second lasing threshold stems from the nonmonotonic dependence of the difference between the modal gain and the internal loss on the level occupancy in a quantumconfined active region [the solid curve in Fig. 1(a)], or, equivalently, on the carrier density in the OCL [the solid curve in Fig. 1(b)], or on the injection current density [the solid curve in Fig. 1(c)]. The point is that the modal gain $g=g^{\max }\left(2 f_{n}-1\right)$ increases linearly with $f_{n}$ [the dotted line in Fig. 1(a)] and saturates at its maximum value $g^{\max }$ as $f_{n} \rightarrow 1$ [which corresponds to $n \rightarrow \infty$ and $j \rightarrow \infty$-see (3), (8) and Fig. 1(b), (c)]. At the same time, $\alpha_{\text {int }}$ is superlinear in $f_{n}$ [see (1) and (3) and the dashed curve in Fig. 1(a)] and increases infinitely as $f_{n} \rightarrow 1$. At a certain $f_{n}$ [see (23)], i.e., at a certain $j$, the rate of increase in $\alpha_{\text {int }}$ with $j$ will inevitably equal that of increase in $g$, and hence the difference $g-\alpha_{\text {int }}$ will peak. 
Any further increase of the injection current density will decrease the difference $g-\alpha_{\text {int }}$ [the solid curve in Fig. 1(c)]. This corresponds to the so-called "loss-multiplication" regime, discussed in [12], [13] for InGaAsP-InP-based strained-layer multiple-QW lasers and attributed to the pileup of carriers due to electrostatic band-profile deformation [17], [18]. In the context of QD lasers, the loss-multiplication regime was discussed in [19] and [20]. As evident from our analysis, this regime and the second lasing threshold are inherent to all structures where the internal loss depends on the carrier density in the OCL.

Due to bimolecular (quadratic in $n$ ) spontaneous recombination in the OCL, the injection current density $j$ is superlinear in $n$ [quadratic at high $n$-see (8)] and hence the internal loss [being linear in $n$-see the dashed curve in Fig. 1(b)] is strongly sublinear in $j$ [increases as $\sqrt{j}$ at high $j$-see the dashed curve in Fig. 1(c)]. [Also the modal gain is strongly sublinear in both $n$ and $j$-see (6), (8), and the dotted curves in Fig. 1(b) and (c)]. In [19], a linear relation between $\alpha_{\text {int }}$ and $j$ was however assumed, which is justified for only monomolecular (linear in $n$ ) recombination in the OCL, such as recombination via nonradiative centers. At high injection levels, bimolecular and then Auger (cubic in $n$ ) recombination dominate and $j$ becomes superlinear in $n$ and hence $\alpha_{\text {int }}$ sublinear in $j$.

\section{Two-VAlUEd CHARACTERISTICS: GAIN-CURRENT AND LIGHT-CURRENT}

Under CW operation, increasing $j$ from zero, one reaches the first lasing threshold $j_{\text {th1 }}$. Above this threshold, the difference between the gain and the internal loss is pinned at the value of the mirror loss $\beta$ and hence Fig. 1 (which is valid for determining the positions of both thresholds) no longer applies. What actually happens above $j_{\text {th1 } 1}$ is shown in Fig. 2, derived [21] by rigorously solving the rate equations in the presence of light generation. These rate equations are presented in Appendix II. In a steady state, the rate equation for photons reduces to our (2), where now the quantities $f_{n}, f_{p}$, and $n$ are calculated in the presence of light generation.

As a consequence of the noninstantaneous carrier capture from the OCL into the quantum-confined active region, the freecarrier density $n$ in the OCL does not pin and increases above threshold. A quantitative theoretical study of this effect was given in [16]. The effect has also been seen experimentally, see [4] and numerous references cited in [16]. To simplify the consideration, the carrier-density-dependent component of the internal loss [the last term in the right-hand side of (2)] was neglected in [16]; with that assumption, the confined-carrier level occupancy $f_{n}$ in the active region is pinned above threshold at a value given by (14), as is evident from (2).

As is also evident from (2), the carrier-density-dependent component of the internal loss in the OCL couples the confined-carrier level occupancy $f_{n}$ in the active region and the free-carrier density $n$ in the OCL; the equation relating these quantities is [we assume equal electron and hole occupancies $\left.\left(f_{n}=f_{p}\right)\right]$

$$
f_{n}=\frac{1}{2}\left(1+\frac{\beta+\alpha_{0}+\sigma_{\mathrm{int}} n}{g^{\max }}\right) .
$$

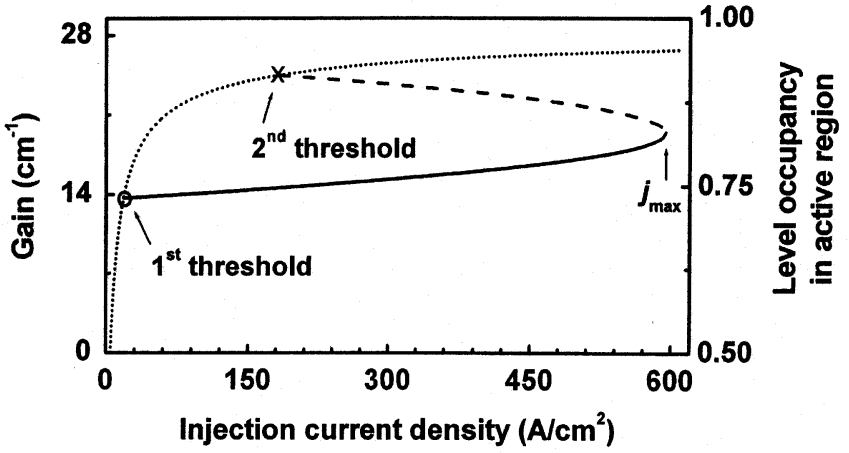

(a)

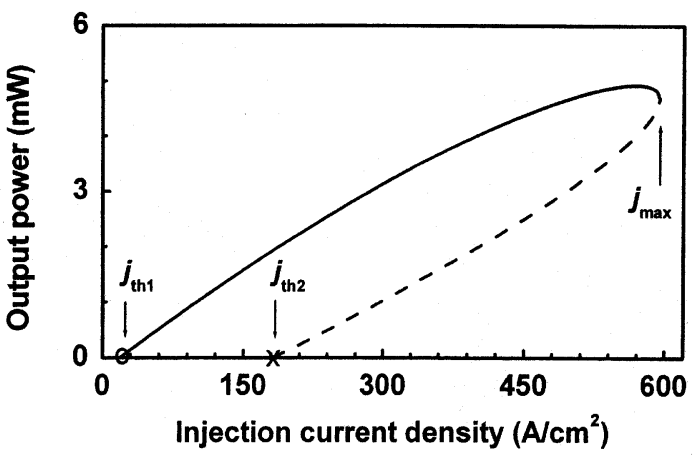

(b)

Fig. 2. Two-valued lasing characteristics: (a) gain-current (left axis) and (b) light-current. The branches corresponding to the first (conventional) and the second (anomalous) regimes (solid and dashed curves, respectively) merge together at the point $j_{\max }$ which defines the maximum operating current. At $j>j_{\max }$, the lasing is quenched. The dotted curve in (a) is the gain-current dependence for a nonlasing regime. Since $g=g^{\max }\left(2 f_{n}-1\right)$, the same curves in (a) show the confined-carrier level occupancy $f_{n}$ in the active region (right axis): solid and dashed curves-for the first and the second lasing regimes, respectively, dotted curve-for a nonlasing regime. The intersections of the solid and dashed curves for the first and the second lasing regimes with the dotted curve for nonlasing regime determine the first and the second lasing thresholds (the abscissae determine $j_{\mathrm{th} 1}$ and $j_{\mathrm{th} 2}$, the ordinates determine $f_{n_{\text {_th1 }}}$ and $\left.f_{n_{-\mathrm{th}} 2}\right)$. In (b), the assumed stripe width $W=2 \mu \mathrm{m}$.

As seen from (15), the carrier-density dependence of the internal loss in the OCL, together with the absence of carrier-density pinning in the OCL in the presence of light generation, leads to nonpinning of the confined-carrier level occupancy $f_{n}$ in the active region in the presence of light generation.

Above the second threshold $j_{\text {th2 }}$ and up to a maximum pump current $j_{\max }$, there are two solutions of the rate equations. The injection-current-density dependence of the confined-carrier level occupancy $f_{n}$ corresponding to the the first solution (conventional lasing regime) and the second solution (anomalous new regime) is shown by the solid and dashed curves, respectively, in Fig. 2(a) (right axis). The intersections of these curves with the dotted curve for $f_{n}$ in the absence of lasing determine the first and the second lasing thresholds (the abscissae determine $j_{\mathrm{th} 1}$ and $j_{\mathrm{th} 2}$, the ordinates determine $f_{n_{-} \text {th } 1}$ and $\left.f_{n \text {-th2 }}\right)$. Since the light intensity is zero at the threshold points, the two solutions for $f_{n}$ of the rate equations in the presence of light generation go (as they should) into $f_{n_{-} \text {th1 }}$ and $f_{n_{-} \text {th2 }}$ determined from (4) and given by (9).

Above the second threshold $j_{\mathrm{th} 2}$, both the gain-current dependence [Fig. 2(a), left axis] and the light-current character- 
istic (LCC) [Fig. 2(b)] are two-valued. At $j=j_{\max }$, the two branches merge in both characteristics.

As seen from (2), in the presence of carrier-density-dependent component of the internal loss the difference between the gain and the internal loss remains pinned at the value of the mirror loss $\beta$, though both the internal loss $\alpha_{\text {int }}=\alpha_{0}+\sigma_{\text {int }} n$ and the gain $g=g^{\max }\left(2 f_{n}-1\right)$ [Fig. 2(a), left axis] change with the injection current. As $\alpha_{\text {int }}$ increases with the current above the conventional threshold $j_{\text {th } 1}$ in the first (conventional) lasing regime, the gain strictly follows it so as to maintain the stable generation condition $g-\alpha_{\text {int }}=\beta$. An increase of $\alpha_{\text {int }}=\alpha_{0}+\sigma_{\text {int }} n$, caused by increasing free-carrier density $n$ in the OCL, is compensated by an increase in $g=g^{\max }\left(2 f_{n}-1\right)$, ensured by increasing confined-carrier level occupancy $f_{n}$ above the conventional threshold in the first lasing regime [the solid curve in Fig. 2(a)]. This continues up to the maximum pump current $j_{\max }$ at which the lasing is quenched.

At this time, we cannot propose a definite experimental technique to access the second lasing regime (the upper branch of the gain-current characteristic [the dashed curve in Fig. 2(a)] and the lower branch of the LCC [the dashed curve in Fig. 2(b)]). Analysis of the stability of the second lasing regime will be published elsewhere. We would like to stress that the nature of the anomalous regime in laser operation deserves further study. In particular, even though this regime is a true steady state, it is not clear whether or not it can be made stable. In some sense, one can draw an analogy between the light-current characteristics of a laser and the current-voltage characteristics of various negative differential resistance devices, such as those based on tunneling or real-space transfer nonlinearities. For example, the numerical study of real-space transfer transistors [22] revealed the existence of multiply connected current-voltage characteristics, with several stable points at a given voltage. In some cases, the anomalous branch may even be unconditionally stable, but even if it is not, its very presence should have a pronounced effect on the large-signal dynamics of laser operation.

Other mechanisms, such as carrier heating and modal gain compression, can also lead to the second lasing threshold. Thus, due to the increase in carrier temperature with the injection current [23]-[26], [18], the modal gain itself can become nonmonotonic with $j$, decreasing at high currents [25]. Such mechanisms can further enhance the effect of internal loss. The effect of internal loss in the presence of other mechanisms is a matter of a separate study. This study will show the relative importance of different mechanisms involved and how to discriminate them from each other. Here, it is however worth noting that the internal loss will remain present in temperature-stabilized devices, in which the heating effects are strongly suppressed.

\section{CRitical Tolerable Parameters}

The lasing in a structure is only possible in a certain region of values of the structure parameters. This multidimensional region of tolerable parameters is given by the existence condition of real positive roots $f_{n_{-} \text {th1 }}$ and $f_{n_{-} \text {th2 }}$ [see (9)] of (4). This condition is of the form

$$
\sqrt{1+\frac{\beta+\alpha_{0}}{g^{\max }}}+\sqrt{\frac{\sigma_{\text {int } n_{1}}}{g^{\max }}} \leq \sqrt{2}
$$

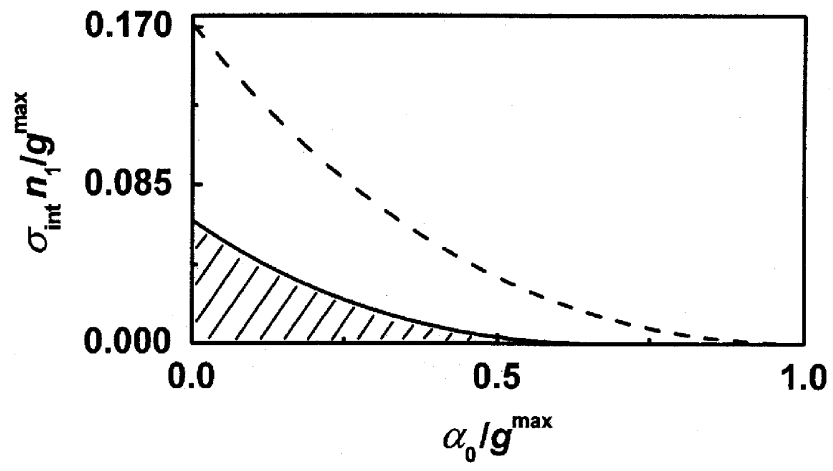

Fig. 3. Two-dimensional region of tolerable values of the normalized internal loss parameters $\alpha_{0} / g^{\max }$ and $\sigma_{\mathrm{int}} n_{1} / g^{\max }$ given by (16) (the hatched region below the solid curve); the ratio $\beta / g^{\max }=0.34$, which corresponds to $\beta=$ $10 \mathrm{~cm}^{-1}$ and $g^{\max }=29.52 \mathrm{~cm}^{-1}$ for the structure considered. The tolerable region for the case $\beta=0$ is the region below the dashed curve. The boundary (the solid or the dashed curve at $\beta / g^{\max }=0.34$ or $\beta / g^{\max }=0$, respectively) represents the maximum tolerable value of $\sigma_{\mathrm{int}}, \sigma_{\mathrm{int}}^{\max }$, versus $\alpha_{0}$; and vice versa, the maximum tolerable value of $\alpha_{0}, \alpha_{0}^{\max }$, versus $\sigma_{\text {int }}$ if the functional relationship between the abscissa and the ordinate is interchanged.

In the absence of internal loss, (16) reduces to the inequality $g^{\max } \geq \beta$ discussed earlier [14], [27].

The limiting case when the inequality (16) becomes an equation yields the critical tolerable value for any one of the parameters, other parameters being fixed. These critical tolerable parameters are $\alpha_{0}^{\max }, \sigma_{\text {int }}^{\max }$ (Section $\mathrm{V}-\mathrm{A}$ ) and $L^{\min }$ (and, equivalently, $\beta^{\max }$ ) (Section V-B). In QD lasers, two more critical parameters are $N_{S}^{\min }$ and $\delta^{\max }$ [14], [27] (Section V-C).

When the equality in (16) holds, there is only one solution of the threshold condition. The curve for $g^{\max }\left(2 f_{n}-1\right)-\alpha_{\text {int }}$ is tangent at its maximum to the horizontal line for the mirror loss $\beta$ (Fig. 1). This happens as $L$ or $\alpha_{0}$ (affecting the constant component of the total loss), or $\sigma_{\text {int }}$ (affecting the carrier-density-dependent component of the internal loss), or, in the context of QD lasers, $N_{S}$ or $\delta$ [affecting $g^{\max }$ —see (20)] tend to their critical tolerable values. In this case,

$$
\begin{aligned}
f_{n_{-} \text {th } 1}=f_{n_{-} \text {th } 2}=f_{n_{-} \text {th }}^{\text {crit }} & =\sqrt{\frac{1}{2}\left(1+\frac{\beta+\alpha_{0}}{g^{\max }}\right)} \\
& =1-\sqrt{\frac{1}{2} \frac{\sigma_{\text {int } n_{1}}}{g^{\max }}}
\end{aligned}
$$

(see (10) for $f_{n_{-} \text {th }}^{\text {crit }}$ ).

\section{A. Critical Tolerable Values of $\alpha_{0}$ and $\sigma_{\mathrm{int}}$}

The loss parameters $\alpha_{0}$ and $\sigma_{\text {int }}$ are not directly controllable variables as they are determined by the specific loss processes involved. Nevertheless, it is instructive to determine the 2-D region of tolerable values of $\alpha_{0}$ and $\sigma_{\text {int }}$ where lasing can be attained (the hatched region in Fig. 3) for given structure parameters. This procedure becomes even more appealing in view of the wide scatter of reported data for $\alpha_{\text {int }}$, even for similar structures. For example, $\alpha_{\text {int }}=1.2 \mathrm{~cm}^{-1}[28]$ and $\alpha_{\text {int }}=11 \mathrm{~cm}^{-1}$ [29] was reported in structures with InGaAs QDs based on GaAs substrates (in the wavelength ranges $\lambda_{0}=1.25-1.29 \mu \mathrm{m}$ and 1-1.1 $\mu \mathrm{m}$, respectively). In [29], the internal loss was unaffected by the number of QD layers, which indicates that the carrier-density-dependent component of $\alpha_{\text {int }}$ was negligible; hence the measured value of $11 \mathrm{~cm}^{-1}$ can be attributed 
solely to $\alpha_{0}$. The estimated value of $\sigma_{\text {int }}$ is $1.3 \times 10^{-17} \mathrm{~cm}^{2}$ in [6] while it is in the range of $2.1 \pm 0.3 \times 10^{-17} \mathrm{~cm}^{2}$ in [10] for GaInAsP-InP double-heterostructure lasing at $\lambda_{0}=1.3 \mu \mathrm{m}$. For GaInAsP-InP double-heterostructure lasing at $\lambda_{0}=1.6 \mu \mathrm{m}, \sigma_{\text {int }}=2.5 \times 10^{-17} \mathrm{~cm}^{2}$ in [6] and $\sigma_{\text {int }}=4 \times 10^{-17} \mathrm{~cm}^{2}$ in [5], [7].

The solid curve [given by the equality in (16)] in Fig. 3 bounds the region of tolerable values of $\alpha_{0}$ and $\sigma_{\text {int }}$ for a given mirror loss $\beta=10 \mathrm{~cm}^{-1}$; the dashed curve is the corresponding upper bound, obtained by assuming an infinitely long cavity $(\beta=0)$. Each point on the solid (dashed) curve presents the maximum tolerable value of $\sigma_{\text {int }}$ at a fixed $\alpha_{0}$ and given $L$ (at $L=\infty$ ); and vice versa, maximum tolerable value of $\alpha_{0}$ at a fixed $\sigma_{\text {int }}$.

At $L=\infty$ and $\alpha_{0}=0$, we have

$$
\sigma_{\text {int }}^{\max }=(3-2 \sqrt{2}) \frac{g^{\max }}{n_{1}} \approx 0.17 \frac{g^{\max }}{n_{1}}
$$

(see the intersection of the dashed curve and the vertical axis in Fig. 3).

$$
\begin{aligned}
& \text { At } L=\infty \text { and } \sigma_{\text {int }}=0 \text {, the equation for } \alpha_{0}^{\max } \text { is } \\
& \alpha_{0}^{\max }=g^{\max }
\end{aligned}
$$

(see the tangent point of the dashed curve and the horizontal axis in Fig. 3).

All of the above equations apply equally to QD, QWR, and QW lasers. One specifies the type of laser by substituting the relevant expression for $g^{\max }$ and relation between $j_{\text {spon }}^{\text {active and }}$ $f_{n}$ [see (8) and (A1)-(A3)].

Our general approach is illustrated below by detailed calculations for QD lasers. The saturation value of the modal gain is given by [14], [30]

$$
g^{\max }=\frac{\xi}{4}\left(\frac{\lambda_{0}}{\sqrt{\epsilon}}\right)^{2} \frac{1}{\tau_{\mathrm{QD}}} \frac{\hbar}{(\Delta \varepsilon)_{\text {inhom }}} \frac{\Gamma}{a} N_{S}
$$

where $\xi=1 / \pi$ and $\xi=1 / \sqrt{2 \pi}$ for the Lorentzian and the Gaussian QD-size distributions, respectively, $\lambda_{0}$ is the lasing wavelength, $\epsilon$ is the dielectric constant of the OCL, $a$ is the mean size of QDs, and $\Gamma$ is the optical confinement factor in a QD layer (along the transverse direction in the waveguide). The inhomogeneous line broadening caused by fluctuations in QD sizes is $(\Delta \varepsilon)_{\text {inhom }}=\left(q_{n} \varepsilon_{n}+q_{p} \varepsilon_{p}\right) \delta$, where $\varepsilon_{n}$ and $\varepsilon_{p}$ are the quantized energy levels of an electron and a hole in a mean-sized QD, $q_{n, p}=-\left(\partial \ln \varepsilon_{n, p} / \partial \ln a\right)$ and $\delta$ is the rms of relative QD size fluctuations.

For illustration, we consider room-temperature operation of a GaInAsP-InP heterostructure similar to that assumed in [14]-[16]. Throughout the paper, we assume the following structure parameters, unless otherwise specified: $\delta=0.05$ (10\% QD-size fluctuations); as-cleaved facet reflectivity at both ends $(R=0.32)$ and $L=1.139 \mathrm{~mm}$, which correspond to the mirror loss $\beta=10 \mathrm{~cm}^{-1} ; N_{S}=6.11 \times 10^{10} \mathrm{~cm}^{-2}$, which, in the absence of internal loss, is the optimum $N_{S}$ minimizing the threshold current density at the above values of $\delta$ and $\beta$ [see the dotted curve in Fig. 7(c)]. At these parameters, $g^{\max }=29.52 \mathrm{~cm}^{-1}$. At $T=300 \mathrm{~K}, n_{1}=5.07 \times 10^{16} \mathrm{~cm}^{-3}$.
For this structure, (18) and (19) give $\sigma_{\text {int }}^{\max }=9.99 \times 10^{-17} \mathrm{~cm}^{2}$ and $\alpha_{0}^{\max }=29.52 \mathrm{~cm}^{-1}$.

We see from (18) to (20) that $\sigma_{\text {int }}^{\max }$ and $\alpha_{0}^{\max }$ increase indefinitely with either $N_{S} \rightarrow \infty$ or $\delta \rightarrow 0$. Hence making the QD ensemble denser or improving the QD-size uniformity is a direct way to alleviate the limitations on lasing imposed by the internal loss in QD structures.

\section{B. Critical Tolerable Values of $L$ and $\beta$}

The minimum cavity length is readily obtained from (11) and (16) and is given by

$$
L^{\min }=\frac{L_{0}^{\min }}{\left(\sqrt{2}-\sqrt{\frac{\sigma_{\text {int }} n_{1}}{g^{\max }}}\right)^{2}-1-\frac{\alpha_{0}}{g^{\max }}}
$$

where $L_{0}^{\min }$ is the minimum cavity length in the absence of internal loss [see (12)].

The equation for the critical tolerable parameters [equality in (16)] can be rewritten as follows:

$$
\beta^{\max }=\left(\sqrt{2 g^{\max }}-\sqrt{\sigma_{\text {int }} n_{1}}\right)^{2}-g^{\max }-\alpha_{0}
$$

where $\beta^{\max }=\left(1 / L_{\min }\right) \ln (1 / R)$ is the maximum tolerable mirror loss. Equation (22) has an evident meaning. The right-hand side is simply the peak value of the difference between the modal gain and the internal loss (Fig. 1); this value is obtained when the level occupancy in the active region is

$$
f_{n}=1-\sqrt{\frac{\sigma_{\text {int }} n_{1}}{2 g^{\max }}}
$$

[see also the last equation in (17)]. When the mirror loss approaches this peak value, the critical condition (22) is met. The peak value of the difference between the modal gain and the internal loss can be considerably lower than the saturation value $g^{\max }$ of the modal gain itself; in addition, in contrast to $g^{\max }$, it is temperature-dependent [through the $T$-dependence of the quantity $n_{1}$ characterizing the intensity of the thermal escape of carriers from an active region, see (3)].

Equations (21)-(23) hold true for QD, QWR, and QW lasers. For QD lasers, using (20) for $g^{\max }$ and (12), we have [27]

$$
L_{0}^{\min }=\frac{4}{\xi}\left(\frac{\sqrt{\epsilon}}{\lambda_{0}}\right)^{2} \tau_{\mathrm{QD}} \frac{a}{\Gamma} \frac{\left(q_{n} \varepsilon_{n}+q_{p} \varepsilon_{p}\right) \delta}{\hbar} \frac{1}{N_{S}} \ln \frac{1}{R}
$$

Fig. 4(a) shows $L^{\mathrm{min}}$ as a function of $\sigma_{\text {int }}$ calculated using (21) and (24). As evident from the figure, depending on $\alpha_{0}$ and $\sigma_{\text {int }}$, the restriction $L^{\min }$ can be considerably increased compared to its value $L_{0}^{\min }$ in the absence of internal loss. This is consistent with the discussion in [19], [20], concerning the limitation of $L^{\mathrm{min}}$ for the QD-ground-state lasing posed by a steep increase in $\alpha_{\text {int }}$ with decreasing cavity length (due to loss-multiplication [12], [13]).

Throughout the paper, we chose $\alpha_{0}=3 \mathrm{~cm}^{-1}$ and $\sigma_{\text {int }}=2.67 \times 10^{-17} \mathrm{~cm}^{2}$ (unless otherwise specified), so that $L^{\min }, \beta^{\max }, N_{S}^{\min }$ and $\delta^{\max }$ are equal to $1.139 \mathrm{~mm}, 10 \mathrm{~cm}^{-1}$, 


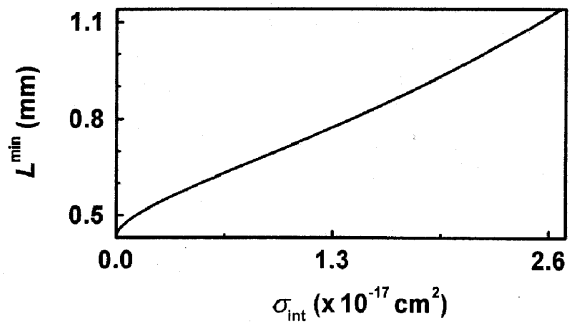

(a)

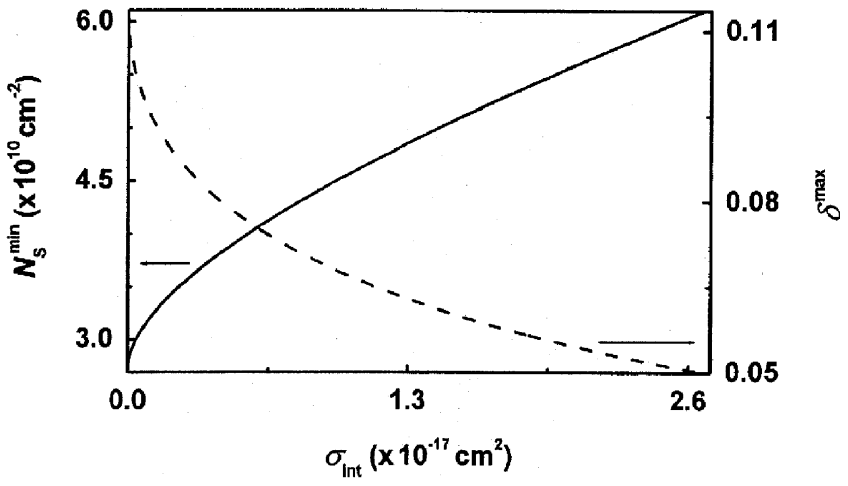

(b)

Fig. 4. (a) Minimum cavity length $L^{\mathrm{min}}$ and (b) minimum surface density of QDs $N_{S}^{\min }$ [solid curve, left axis] and maximum rms of relative QD size fluctuations $\delta^{\max }$ [dashed curve, right axis] against absorption loss cross section $\sigma_{\text {int }} . L^{\min }, N_{S}^{\min }$, and $\delta^{\max }$ are calculated using (21), (26), and (27), respectively. The same curves can be viewed as representing $\sigma_{\text {int }}^{\max }$ versus the cavity length $L$, the surface density $N_{S}$ of QDs, or the rms $\delta$ of relative QD size fluctuations.

$6.11 \times 10^{10} \mathrm{~cm}^{-2}$ and 0.05 , respectively. At these plausible $\alpha_{0}$ and $\sigma_{\text {int }}$, the internal loss is within a typical range from several to above ten $\mathrm{cm}^{-1}$ (the solid curve and the left axis in Fig. 8). The minimum cavity length is hence increased almost threefold compared to its value in the absence of internal loss $L_{0}^{\min }=386 \mu \mathrm{m}$. Thus, our theory shows that the absence of lasing often observed in short-cavity QD structures can be attributed to internal loss. Another possible reason that limits lasing via the ground-state transition at short (under a millimeter) cavity lengths can be a small overlap integral of the electron and hole wave functions in low-symmetry QDs; this was discussed in [31].

When the denominator of the right-hand side in (21) is zero, then $L^{\min } \rightarrow \infty$, i.e., the lasing is unattainable at a finite cavity length. This situation at a high internal loss may be somewhat alleviated by using high-reflectivity mirrors. Indeed, when $R \rightarrow$ 1 , then $L_{0}^{\mathrm{min}} \rightarrow 0$ [see (24)] and $L^{\mathrm{min}}$ can be kept finite.

\section{Critical Tolerable Values of $N_{S}$ and $\delta$}

The equation for the critical tolerable parameters can be put in the following form (applicable equally to QD, QWR, and QW lasers):

$$
g^{\max }=\left(\sqrt{\beta+\alpha_{0}+\sigma_{\text {int }} n_{1}}+\sqrt{2 \sigma_{\text {int }} n_{1}}\right)^{2} .
$$

In the absence of internal loss, we obtain the earlier equation $g^{\max }=\beta$ [27]. With internal loss, the role of the mirror loss $\beta$ is effectively taken on by the right-hand side of (25).

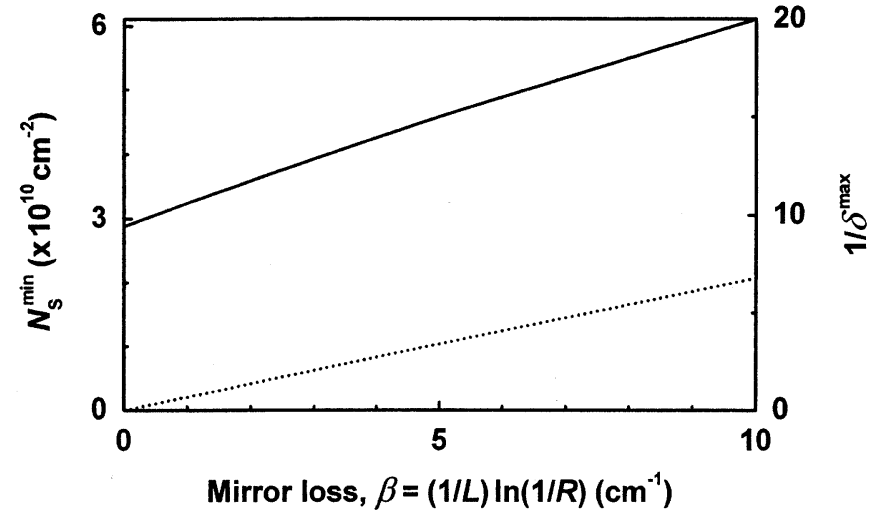

Fig. 5. Minimum surface density of QDs $N_{S}^{\min }$ (left axis) and reciprocal of maximum rms of relative QD size fluctuations $1 / \delta^{\max }$ (right axis) against mirror loss $\beta$ [see (26) and (27)]. The dotted line corresponds to the case of no internal loss [see (28) and (29)]. The same curve can be viewed as representing $\beta^{\max }$ versus the surface density $N_{S}$ of QDs or the reciprocal of the rms of relative QD size fluctuations $1 / \delta$.

Using (20) to express $g^{\max }$ of a QD laser in terms of $N_{S}$ and $\delta$, we get the critical tolerable values for $N_{S}$ and $\delta$ from (25) as follows:

$$
\begin{aligned}
& N_{S}^{\min }=N_{S 0}^{\min }\left(\sqrt{1+\frac{\alpha_{0}+\sigma_{\text {int }} n_{1}}{\beta}}+\sqrt{\frac{2 \sigma_{\text {int }} n_{1}}{\beta}}\right)^{2} \\
& \delta^{\max }=\frac{\delta_{0}^{\max }}{\left(\sqrt{1+\frac{\alpha_{0}+\sigma_{\text {int }} n_{1}}{\beta}}+\sqrt{\frac{2 \sigma_{\text {int }} n_{1}}{\beta}}\right)^{2}}
\end{aligned}
$$

where $N_{S 0}^{\min }$ and $\delta_{0}^{\max }$ are the critical tolerable values in the absence of internal loss [14], [27], given as follows:

$$
\begin{aligned}
N_{S 0}^{\min } & =\frac{4}{\xi}\left(\frac{\sqrt{\epsilon}}{\lambda_{0}}\right)^{2} \tau_{\mathrm{QD}} \frac{\left(q_{n} \varepsilon_{n}+q_{p} \varepsilon_{p}\right) \delta}{\hbar} \beta \frac{a}{\Gamma} \\
\delta_{0}^{\max } & =\frac{\xi}{4}\left(\frac{\lambda_{0}}{\sqrt{\epsilon}}\right)^{2} \frac{1}{\tau_{\mathrm{QD}}} \frac{\Gamma}{a} \frac{\hbar}{\left(q_{n} \varepsilon_{n}+q_{p} \varepsilon_{p}\right)} \frac{N_{S}}{\beta} .
\end{aligned}
$$

Fig. 4(b) shows $N_{S}^{\min }$ and $\delta^{\max }$ against $\sigma_{\text {int }}$ calculated using (26) and (27), respectively. We see that both $N_{S}^{\min }$ and $\delta^{\max }$ are strongly affected by the internal loss: $N_{S}^{\mathrm{min}}$ is increased and $\delta^{\max }$ is decreased.

As $L \rightarrow \infty(\beta \rightarrow 0)$, in contrast to the case of no internal loss (the dotted line in Fig. 5), $N_{S}^{\min }$ does not vanish and $\delta^{\max }$ is finite (the solid line in Fig. 5); in this case, $N_{S}^{\min }$ and $\delta^{\max }$ are solely controlled by $\alpha_{0}$ and $\sigma_{\text {int }}$ being given by (28) and (29) wherein $\beta$ is replaced by $\left(\sqrt{\alpha_{0}+\sigma_{\text {int }} n_{1}}+\sqrt{2 \sigma_{\text {int }} n_{1}}\right)^{2}$.

\section{Threshold CurRent Densities Against Structure PARAMETERS}

The confined carrier level occupancies in the active region at both the lower and the upper lasing thresholds, $f_{n_{-} \text {th1 }}$ and $f_{n-\text { th2 }}$, calculated using (9) are shown in Fig. 6 (solid and dashed curves, respectively). The lower and the upper threshold current

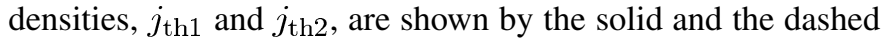
curves, respectively, in Fig. 7. To illustrate how strong the effect of internal loss can be, the level occupancy and the threshold 


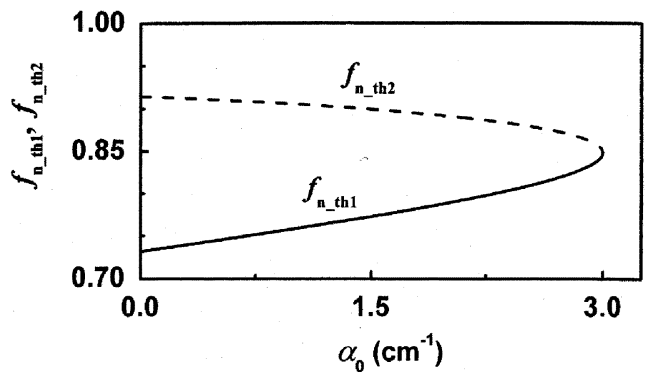

(a)

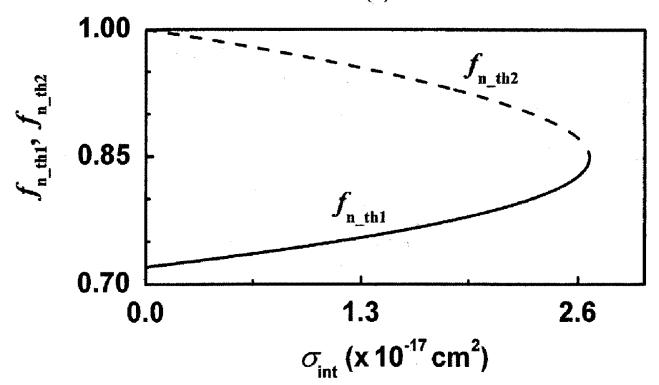

(b)

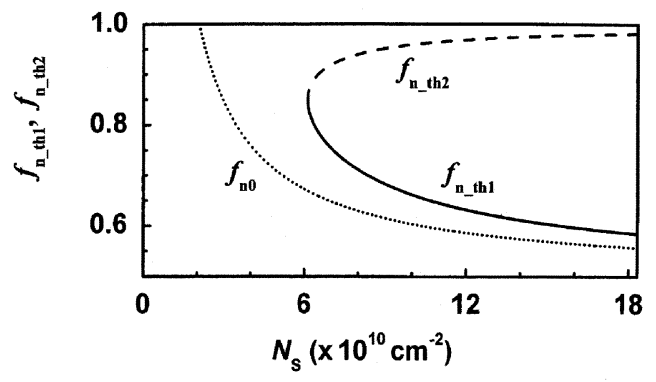

(c)

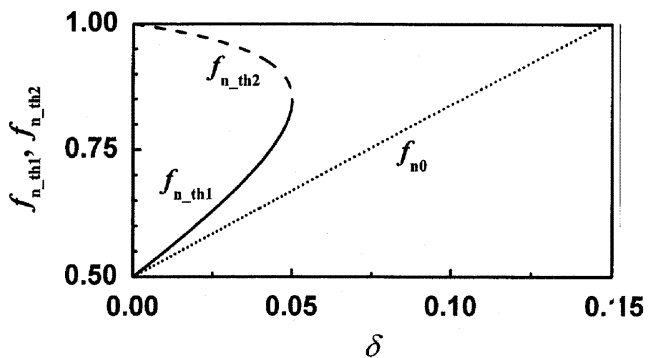

(d)

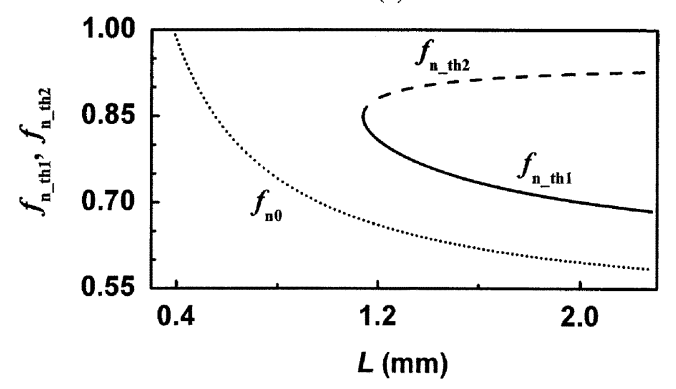

(e)

Fig. 6. Confined-carrier level occupancy in the active region at the lower (solid curves) and upper (dashed curves) lasing thresholds, $f_{n \_ \text {th1 }}$ and $f_{n_{-} \text {th2 }}$ [see (9)], against (a) $\alpha_{0}$, (b) $\sigma_{\text {int }}$, (c) $N_{S}$, (d) $\delta$, and (e) $L$. The dotted curves in (c)-(e) show the level occupancy $f_{n 0}$ at the lasing threshold in the absence of internal loss.

current density in the absence of internal loss, $f_{n 0}$ and $j_{\text {th0 }}$, respectively, are also shown in Figs. 6 and 7 (dotted curves).

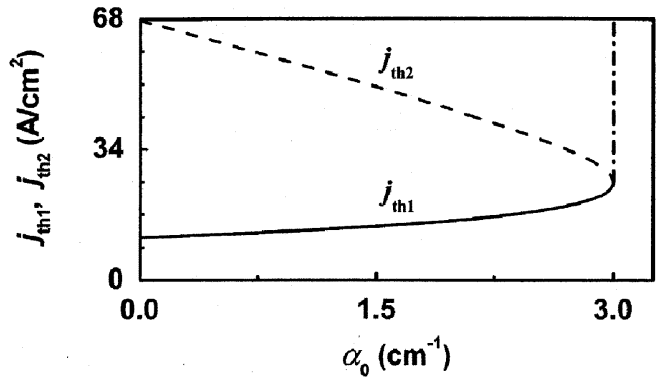

(a)

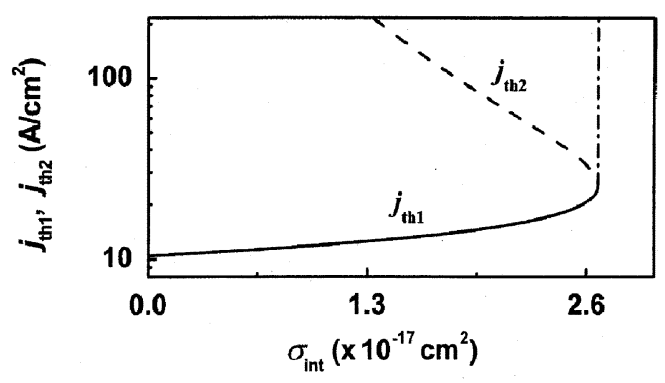

(b)

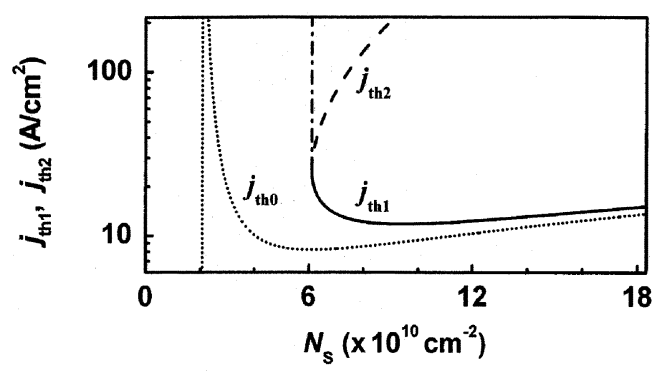

(c)

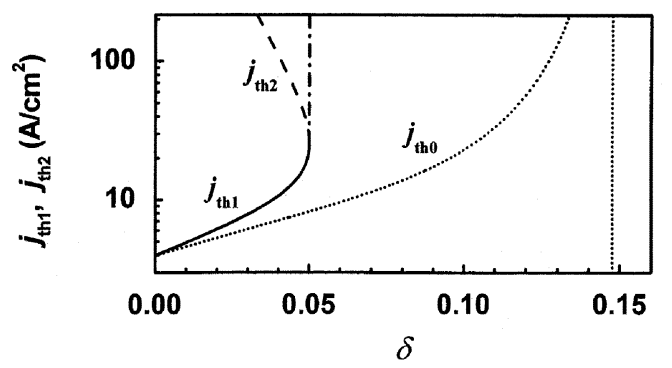

(d)

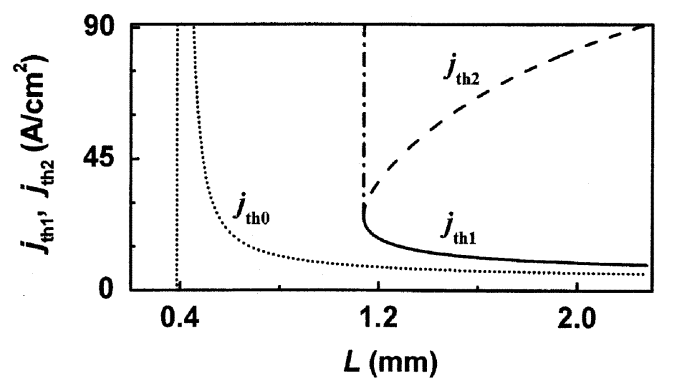

(e)

Fig. 7. Lower and upper threshold current densities (solid and dashed curves, respectively), $j_{\text {th } 1}$ and $j_{\text {th2 }}$, against (a) $\alpha_{0}$, (b) $\sigma_{\text {int }}$, (c) $N_{S}$, (d) $\delta$, and (e) $L$. In (b)-(d), the $y$ axis is shown in the log-scale since $j_{\text {th2 } 2}$ increases rapidly away from the critical point. The curve for $j_{\text {th1 } 1}$ joins smoothly the vertical dash-dotted line at the critical point. The dotted curves and the vertical dotted lines in (c)-(e) show the threshold current density $j_{\text {tho }}$ and its asymptote at the critical point in the absence of internal loss.

In the absence of internal loss, the level occupancy in a quantum-confined active region tends to unity $\left(f_{n 0} \rightarrow 1\right)$ 
when any structure parameter approaches its critical tolerable value [see (11), the dotted curves in Fig. 6(c) and (e) and the inclined dotted line in Fig. 6(d)]; hence, the threshold current density in the absence of internal loss increases infinitely $\left(j_{\text {th } 0} \rightarrow \infty\right)$-see the dotted curves in Fig. 7(c)-(e).

As the structure parameter equals its critical tolerable value in the presence of carrier-density-dependent internal loss $\left(\sigma_{\text {int }} \neq 0\right)$, the two solutions of the threshold condition (the solid and the dashed curves in Fig. 6) merge together at a value given by (17). Hence the lower threshold current density $j_{\text {th1 }}$ (the solid curve in Fig. 7) and the upper threshold current density $j_{\text {th2 }}$ (the dashed curve in Fig. 7) merge together at a finite value. The derivatives of $f_{n}$, and, hence, of $n$ and $j_{\text {th }}$, with respect to the structure parameter are infinitely high at a critical point (Figs. 6-8). This is a consequence of $\partial\left(g-\alpha_{\text {int }}\right) / \partial f_{n}=0$ at this point-see Fig. 1. Immediately behind the critical point, the lasing is unattainable. Hence, the curve for $j_{\text {th } 1}$ joins smoothly the vertical line at the critical point (Fig. 7). In contrast, when only the constant component of the internal loss is present $\left(\sigma_{\text {int }}=0\right)$, the curve for $j_{\text {th } 1}$ approaches only asymptotically the vertical line at the critical point, much as the curve for $j_{\text {tho }}$ does [dotted curves in Fig. 7(c)-(e)].

It is evident from Fig. 7 that the internal loss can have a strong effect on the lower threshold current density $j_{\text {th1 }}$, especially near the critical point, when $j_{\text {th1 }}$ may increase by several times compared to its value $j_{\text {tho }}$ in the absence of internal loss.

The optimum surface density of QD's, minimizing $j_{\text {th1 } 1}$, is $9.39 \times 10^{10} \mathrm{~cm}^{-2}$ [see the solid curve in Fig. 7(c)], i.e., it increases by more than $50 \%$ compared to its value $6.11 \times 10^{10} \mathrm{~cm}^{-2}$ in the absence of $\alpha_{\text {int }}$ [see the dotted curve in Fig. 7(c)].

Fig. 8 shows the free-carrier density in the OCL (right axis) and the internal loss (solid curves, left axis) at the lower lasing threshold against $N_{S}$ (a), $\delta(\mathrm{b})$, and $L$ (c). The dotted curves show the free-carrier density in the OCL in the absence of internal loss. As seen from the figure, the free-carrier density can be considerably increased due to the internal loss. A decrease of $\alpha_{\text {int }}$ with $N_{S}$ [Fig. 8(a)] is in line with the data extracted by the fitting procedure in [19, Fig. 2].

\section{CONCLUSION}

We have carried out a theoretical analysis of the threshold behavior of semiconductor lasers with a reduced-dimensionality active region taking a general account of the internal optical loss.

In any structure where the free-carrier density in the OCL does not pin in the presence of light generation and the internal loss depends on this density, we predict the existence of a second (upper) lasing threshold. Above the second threshold, there exist two physically distinct steady-state regimes of stimulated emission. Accordingly, the gain-current characteristic and the LCC are two-valued up to a maximum current at which the lasing is quenched.

Due to the internal loss, the region of tolerable values of the structure parameters is strongly narrowed, and both the freecarrier density outside the active region and the confined-carrier

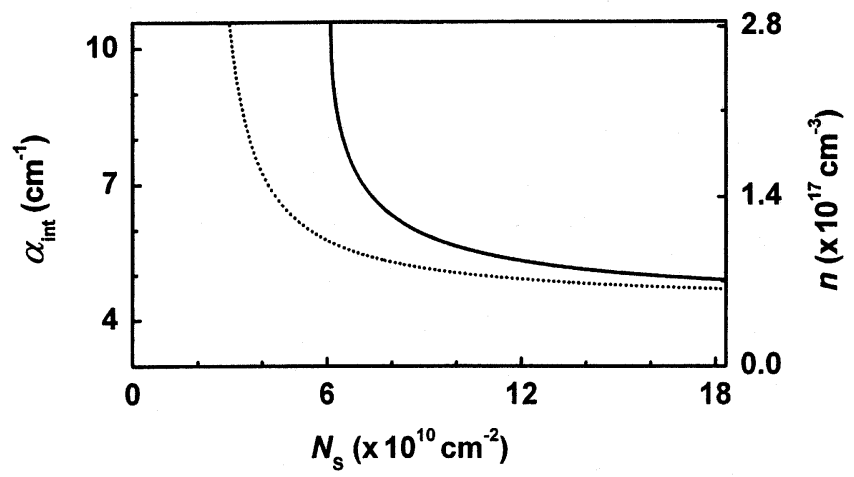

(a)

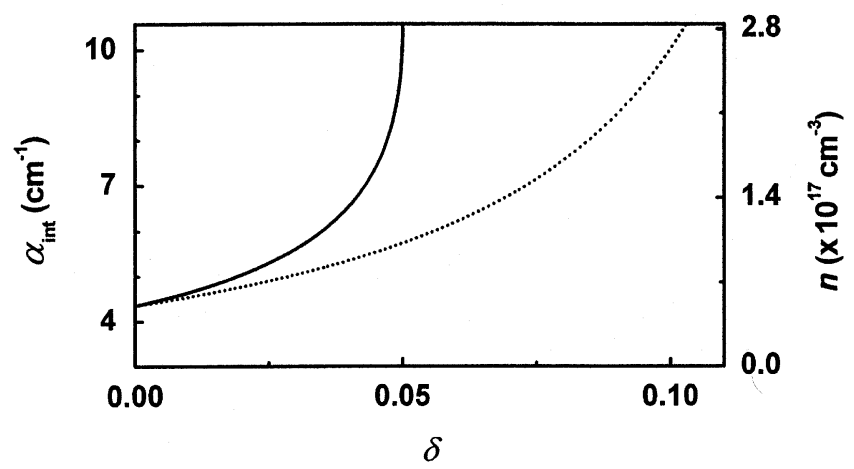

(b)

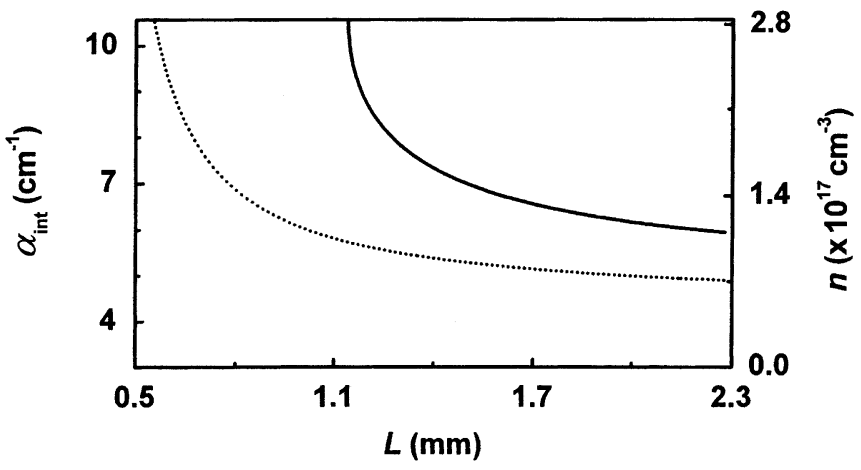

(c)

Fig. 8. Free-carrier density in the OCL (right axis) and internal loss (left axis) at the lower lasing threshold against (a) $N_{S}$, (b) $\delta$, and (c) $L$. The dotted curves show $n$ in the absence of internal loss.

level occupancy in the active region at the lasing threshold are increased; thus the threshold current density is increased.

Presented analysis, exemplified in the context of QD lasers, can be used for their further optimizing, especially for lowering the threshold current density in short-cavity structures.

\section{APPENDIX I}

SPONTANEOUS RECOMBINATION CURRENT DENSITY EXPRESSED IN TERMS OF THE LEVEL OCCUPANCY IN A QUANTUM-CONFINED ACTIVE REGION

For QD-, QWR-, and QW-active regions, we have, respectively [14], [16]

$$
j_{\mathrm{spon}}^{\mathrm{QD}}=\frac{e N_{S}}{\tau_{\mathrm{QD}}} f_{n}^{2}
$$


where $N_{S}$ is the surface density of QDs and $\tau_{\mathrm{QD}}$ is the spontaneous radiative lifetime in a $\mathrm{QD}$

$$
j_{\mathrm{spon}}^{\mathrm{QWR}}=e N_{L} B_{1 D} n_{1 D}^{2}\left(f_{n}\right)
$$

where $N_{L}$ is the linear density of QWRs (in $\mathrm{cm}^{-1}$ ) and $B_{1 D}$ is the radiative constant for a 1-D region (in $\mathrm{cm} / \mathrm{s}$ ); and

$$
j_{\mathrm{spon}}^{\mathrm{QW}}=e N_{\mathrm{QW}} B_{2 D} n_{2 D}^{2}\left(f_{n}\right)
$$

where $N_{\mathrm{QW}}$ is the number of QWs and $B_{2 D}$ is the radiative constant for a 2-D region (in $\mathrm{cm}^{2} / \mathrm{s}$ ).

The 2-D carrier density in a QW is expressed in terms of $f_{n}$ as follows [32]:

$$
n_{2 D}=N_{c}^{2 D} \ln \frac{1}{1-f_{n}}
$$

where $N_{c}^{2 D}=m_{c}^{\mathrm{QW}} T / \pi \hbar^{2}$.

A functional relationship between the 1-D carrier density in a QWR and $f_{n}$ is also readily calculated, although a closed-form expression may be difficult to derive in the general case.

\section{APPENDIX II \\ Rate EQuations in the PRESENCE OF Light GenERation}

We shall confine ourselves to the case of a QD laser, for which we have performed all illustrative calculations in this paper. In this case, the rate equations are of the following forms.

For carriers confined in a QD (assuming $f_{n}=f_{p}$ ), we have

$$
\begin{aligned}
\frac{\partial f_{n}}{\partial t}= & \sigma_{n} v_{n} n\left(1-f_{n}\right) \\
& -\sigma_{n} v_{n} n_{1} f_{n}-\frac{f_{n}^{2}}{\tau_{\mathrm{QD}}}-\frac{c}{\sqrt{\epsilon_{g}}} \frac{g^{\max }}{N_{S} S}\left(2 f_{n}-1\right) N .
\end{aligned}
$$

For free carriers in the OCL (assuming $n=p$ ), we have

$$
\frac{\partial n}{\partial t}=\sigma_{n} v_{n} n_{1} \frac{N_{S}}{b} f_{n}-\sigma_{n} v_{n} n \frac{N_{S}}{b}\left(1-f_{n}\right)-B n^{2}+\frac{j}{e b} .
$$

For photons, we have

$$
\frac{\partial N}{\partial t}=\frac{c}{\sqrt{\epsilon_{g}}} g^{\max }\left(2 f_{n}-1\right) N-\frac{c}{\sqrt{\epsilon_{g}}}\left(\beta+\alpha_{\mathrm{int}}\right) N .
$$

In (A5)-(A7), $\sigma_{n}$ is the cross section of carrier capture into a $\mathrm{QD}, v_{n}$ is the carrier thermal velocity, $c$ is the light velocity in vacuum, $\sqrt{\epsilon_{g}}$ is the group index of the dispersive OCL material, $S=W L$ is the QD layer area (the cross section of the junction), $W$ is the QD layer width (the lateral size of the device), and $N$ is the number of photons in the lasing mode.

In a steady state, (A7) reduces to (2). We thus see that (2), which is commonly used to present the lasing threshold condition, retains the same form above threshold, in the presence of light generation. It should be noted, however, that the quantities $f_{n}$ and $n$ must now be computed self-consistently, from the full set of steady-state rate equations in the presence of light generation.
For the general case, applicable to semiconductor lasers with a quantum-confined active region of arbitrary dimensionality, the rate equations can be found in [16].

\section{REFERENCES}

[1] G. P. Agrawal and N. K. Dutta, Long-Wavelength Semiconductor Lasers. New York: Van Nostrand, 1986, p. 474.

[2] Quantum Well Lasers, P. S. Zory Jr., Ed., Academic, Boston, MA, 1993, p. 504.

[3] L. A. Coldren and S. W. Corzine, Diode Lasers and Photonic Integrated Circuits. New York: Wiley, 1995, p. 594.

[4] D. Z. Garbuzov, A. V. Ovchinnikov, N. A. Pikhtin, Z. N. Sokolova, I. S. Tarasov, and V. B. Khalfin, "Experimental and theoretical investigations of singularities of the threshold and power characteristics of InGaAsP/InP separate-confinement double-heterostructure lasers $(\lambda=$ $1.3 \mu \mathrm{m})$," Sov. Phys. Semicond., vol. 25, no. 5, pp. 560-564, May 1991.

[5] M. Asada, A. R. Adams, K. E. Stubkjaer, Y. Suematsu, Y. Itaya, and S. Arai, "The temperature dependence of the threshold current of GaInAsPAnP DH lasers," IEEE J. Quantum Electron., vol. QE-17, pp. 611-619, May 1981.

[6] C. H. Henry, R. A. Logan, F. R. Merritt, and J. P. Luongo, "The effect of intervalence band absorption on the thermal behavior of InGaAsP lasers," IEEE J. Quantum Electron., vol. QE-19, pp. 947-952, June 1983.

[7] M. Asada, A. Kameyama, and Y. Suematsu, "Gain and intervalence band absorption in quantum-well lasers," IEEE J. Quantum Electron., vol. QE-20, pp. 745-753, July 1984.

[8] N. A. Gun'ko, V. B. Khalfin, Z. N. Sokolova, and G. G. Zegrya, "Optical loss in InAs-based long-wavelength lasers," J. Appl. Phys., vol. 84, no. 1, pp. 547-554, July 1998.

[9] J. J. Lee, L. J. Mawst, and D. Botez, "MOCVD growth of asymmetric $980 \mathrm{~nm} \mathrm{InGaAs/InGaAsP}$ broad-waveguide diode lasers for high power applications," J. Cryst. Growth, vol. 249, no. 1-2, pp. 100-105, Feb. 2003.

[10] D. A. Ackerman, G. E. Shtengel, M. S. Hybertsen, P. A. Morton, R. F. Kazarinov, T. Tanbun-Ek, and R. A. Logan, "Analysis of gain in determining $T_{0}$ in $1.3 \mu \mathrm{m}$ semiconductor lasers," IEEE J. Select. Topics Quantum Electron., vol. 1, pp. 250-63, June 1995.

[11] G. L. Belenky, C. L. Reynolds, D. V. Donetsky, G. E. Shtengel, M. S. Hybertsen, M. A. Alam, G. A. Baraff, R. K. Smith, R. F. Kazarinov, J. Winn, and L. E. Smith, "Role of p-doping profile and regrowth on the static characteristics of 1.3- $\mu \mathrm{m}$ MQW InGaAsP-InP lasers: Experiment and modeling," IEEE J. Quantum Electron., vol. 35, pp. 1515-1520, Oct. 1999.

[12] S. Seki, H. Oohasi, H. Sugiura, T. Hirono, and K. Yokoyama, "Dominant mechanisms for the temperature sensitivity of $1.3 \mu \mathrm{m} \mathrm{InP-based}$ strained-layer multiple-quantum-well lasers," Appl. Phys. Lett., vol. 67, no. 8, pp. 1054-1056, Aug. 1995.

[13] — "Dominant mechanism for limiting the maximum operating temperature of InP-based multiple-quantum-well lasers," J. Appl. Phys., vol. 79, no. 5, pp. 2192-2197, Mar. 1996.

[14] L. V. Asryan and R. A. Suris, "Inhomogeneous line broadening and the threshold current density of a semiconductor quantum dot laser," Semicond. Sci. Technol., vol. 11, no. 4, pp. 554-567, Apr. 1996.

[15] — "Carrier photoexcitation from levels in quantum dots to states of the continuum in lasing," Semicond., vol. 35, no. 3, pp. 343-346, Mar. 2001.

[16] L. V. Asryan, S. Luryi, and R. A. Suris, "Internal efficiency of semiconductor lasers with a quantum-confined active region," IEEE J. Quantum Electron., vol. 39, pp. 404-418, Mar. 2003.

[17] S. Seki and K. Yokoyama, "Electrostatic deformation in band profiles of InP-based strained-layer quantum-well lasers," J. Appl. Phys., vol. 77, no. 10, pp. 5180-5184, May 1995.

[18] L. V. Asryan, N. A. Gun'ko, A. S. Polkovnikov, G. G. Zegrya, R. A. Suris, P.-K. Lau, and T. Makino, "Threshold characteristics of InGaAsP/InP multiple quantum well lasers," Semicond. Sci. Technol., vol. 15 , no. 12 , pp. $1131-1140$, Dec. 2000.

[19] A. E. Zhukov, A. R. Kovsh, V. M. Ustinov, and Z. I. Alferov, "Loss multiplication in a quantum dot laser," Laser Phys., vol. 13, no. 3, pp. 319-323, Mar. 2003.

[20] A. R. Kovsh, N. A. Maleev, A. E. Zhukov, S. S. Mikhrin, A. P. Vasil'ev, E. A. Semenova, Y. M. Shernyakov, M. V. Maximov, D. A. Livshits, V. M. Ustinov, N. N. Ledentsov, D. Bimberg, and Z. I. Alferov, "InAs/In$\mathrm{GaAs} / \mathrm{GaAs}$ quantum dot lasers of $1.3 \mu \mathrm{m}$ range with enhanced optical gain," J. Cryst. Growth, vol. 251, no. 1-4, pp. 729-736, Apr. 2003. 
[21] L. V. Asryan and S. Luryi, "Two lasing thresholds in semiconductor lasers with a quantum-confined active region," Appl. Phys. Lett., vol. 83, no. 26, pp. 5368-5370, Dec. 2003.

[22] S. Luryi and M. R. Pinto, "Broken symmetry and the formation of hotelectron domains in real-space-transfer transistors," Phys. Rev. Lett., vol. 67, no. 17, pp. 2351-2354, Oct. 1991.

[23] P. G. Eliseev, Introduction to the Physics of Injection Lasers (in Russian). Moscow, U.S.S.R.: Nauka, 1983, p. 294.

[24] V. B. Gorfinkel, S. Luryi, and B. Gelmont, "Theory of gain spectra for quantum cascade lasers and temperature dependence of their characteristics at low and moderate carrier concentrations," IEEE J. Quantum Electron., vol. 32, pp. 1995-2003, Nov. 1996.

[25] V. Gorfinkel, M. Kisin, and S. Luryi, "Hot electrons and curves of constant gain in long wavelength quantum well lasers," Opt. Exp., vol. 2, no. 4, pp. 125-130, Feb. 1998.

[26] L. V. Asryan, N. A. Gun'ko, A. S. Polkovnikov, R. A. Suris, G. G. Zegrya, B. B. Elenkrig, S. Smetona, J. G. Simmons, P.-K. Lau, and T Makino, "High-power and high-temperature operation of InGaAsP/InP multiple quantum well lasers," Semicond. Sci. Technol., vol. 14, no. 12, pp. 1069-1075, Dec. 1999

[27] L. V. Asryan and R. A. Suris, "Longitudinal spatial hole burning in a quantum-dot laser," IEEE J. Quantum Electron., vol. 36, pp. 1151-1160, Oct. 2000.

[28] S. S. Mikhrin, A. E. Zhukov, A. R. Kovsh, N. A. Maleev, A. P. Vasil'ev, E. S. Semenova, V. M. Ustinov, M. M. Kulagina, E. V. Nikitina, I. P. Soshnikov, Y. M. Shernyakov, D. A. Livshits, N. V. Kryjanovskaya, D. S. Sizov, M. V. Maksimov, A. F. Tsatsul'nikov, N. N. Ledentsov, D. Bimberg, and Z. I. Alferov, "High efficiency $\left(\eta_{D}>80 \%\right)$ long wavelength $(\lambda>1.25 \mu \mathrm{m})$ quantum dot diode lasers on GaAs substrates," Semicond., vol. 36, no. 11, pp. 1315-1321, Nov. 2002

[29] P. M. Smowton, E. Herrmann, Y. Ning, H. D. Summers, P. Blood, and M Hopkinson, "Optical mode loss and gain of multiple-layer quantum-dot lasers," Appl. Phys. Lett., vol. 78, no. 18, pp. 2629-2631, Apr. 2001.

[30] L. V. Asryan and R. A. Suris, "Charge neutrality violation in quantum dot lasers," IEEE J. Select. Topics Quantum Electron., vol. 3, pp. 148-157, Apr. 1997.

[31] L. V. Asryan, M. Grundmann, N. N. Ledentsov, O. Stier, R. A. Suris, and D. Bimberg, "Effect of excited-state transitions on the threshold characteristics of a quantum dot laser," IEEE J. Quantum Electron., vol. 37, pp. 418-425, Mar. 2001.

[32] K. J. Vahala and C. E. Zah, "Effect of doping on the optical gain and the spontaneous noise enhancement factor in quantum well amplifiers and lasers studied by simple analytical expressions," Appl. Phys. Lett., vol 52, no. 23, pp. 1945-1947, June 1988.
Levon V. Asryan received the M.Sc. degree in radiophysics and electronics from Yerevan State University, Yerevan, Armenia, in 1985 and the Ph.D. degree in physics and mathematics and the Doctor of Physical and Mathematical Sciences degree from the Ioffe Physico-Technical Institute, St. Petersburg, Russia, in 1988 and 2002, respectively.

Since 1992, he has been a Member of Scientific Staff with the Ioffe Institute (since 1999 as a Senior Member). He is currently a Research Associate Professor with the Department of Electrical and Computer Engineering, State University of New York at Stony Brook, on leave from the Ioffe Institute. His research interests include the physics of semiconductors and semiconductor devices, nanoelectronics, optoelectronics, and photonics.

Dr. Asryan was awarded the State Prize of Russia (the highest Russian scientific award) and the first IEEE JOURNAL OF QUANTUM ELECTRONICS Best Paper Award in 2001 for his work on quantum-dot lasers.

Serge Luryi (M'81-SM'85-F'90) received the Ph.D. degree in physics from the University of Toronto, Toronto, ON, Canada, in 1978. His doctoral thesis was devoted to quantum mechanics of intermolecular interactions in solid hydrogen.

In 1980, he joined Bell Laboratories, Murray Hill, NJ, and became interested in the physics and technology of semiconductor devices. In 1994, he joined the State University of New York at Stony Brook, where he is currently a Distinguished Professor and Chair of Electrical and Computer Engineering. He is also Director of the NY State Center for Advanced Sensor Technology. He has published over 180 scientific papers and holds 34 patents.

Dr. Luryi served as the Editor of the IEEE TRANSACTIONS ON ELECTRON DEVICES during 1986-1990. He was elected Fellow of the IEEE for contributions in the field of heterojunction devices in 1989, received the Distinguished Member of Technical Staff Award from Bell Laboratories in 1990, and was elected Fellow of the American Physical Society in 1993 for contributions to the theory of electron transport in low-dimensional systems and invention of novel electron devices. In 1995, he organized an advanced research workshop on the "Future Trends in Microelectronics: Reflections on the Road to Nanotechnology," which grew into a regular series. The fourth workshop in this series, "The Nano..., and the Bio," took place in June 2003 on the island of Corsica. 